\title{
Temporomandibular joint ankylosis: Late presentation, report of 2 cases and review of literature
}

\author{
Adekunle Moses Adetayo, ${ }^{1,2}$ Olanike Abosede Oladapo, ${ }^{2}$ Abdurrazaq Olanrewaju Taiwo, ${ }^{3}$ \\ Ayodele Moses Akinola, ${ }^{1,5}$ Ayodeji Titus Oyedele, ${ }^{1,2}$ Modupe Olusola Adetayo ${ }^{4}$ \\ ${ }^{1}$ Department of Surgery, Benjamin Carson Snr School of medicine, Babcock University, Ilisan-Remo, Ogun \\ State; ${ }^{2}$ Dental Unit, Babcock University Teaching Hospital, Ilisan-Remo, Ogun State; ${ }^{3}$ Department of \\ Surgery, Usmanu DanFodiyo University Sokoto, Sokoto State; ${ }^{4}$ Department of Biochemistry, Babcock \\ University, Ilisan-Remo; ${ }^{5}$ Otolaryngology Unit, Babcock University Teaching Hospital, Ilisan-Remo, Ogun \\ State, Nigeria
}

\begin{abstract}
The Temporomandibular Joint (TMJ) is a unique joint because both the right and left joints must open synchronously for function, Ankylosis of the TMJ refers to bony or fibrous adhesion of the anatomical components of the joint and their ensuing loss of function. Early presentation means that the patient will require a less extensive surgical treatment. The reality, however, is that many patients present to the surgeons, years or decades after the initial injury, and so TMJ ankylosis would have been fully established. Causes of TMJ ankylosis include trauma and infections. Surgery
\end{abstract}

Correspondence: Adekunle Moses Adetayo, Department of Surgery, Benjamin Carson Snr School of medicine, Babcock University, IlisanRemo, Ogun State; Dental Unit, Babcock University Teaching Hospital, Ilisan-Remo, Ogun State, Nigeria.

Tel.: +2348038337845

E-mail: adetayoa@babcock.edu.ng

Key words: TMJ injury; TMJ ankylosis; Delayed treatments.

Conflict of interest: The authors declare no conflict of interest.

Acknowledgement: Our appreciation goes to the staff ad members of the Departments of Otolaryngology and Dentistry for their immense support to the successful treatment of these patients.

Contributions: The manuscript has been read and approved by all authors. The authors contributed equally to the writing of the manuscript.

Funding: None.

Consent for publication: Consent was gotten from the patients.

Received for publication: 10 September 2020.

Revision received: 15 October 2020.

Accepted for publication: 24 October 2020 .

This work is licensed under a Creative Commons Attribution NonCommercial 4.0 License (CC BY-NC 4.0).

${ }^{(C)}$ Copyright: the Author(s),2020

Licensee PAGEPress, Italy

Annals of Clinical and Biomedical Research 2020; 1:106

doi:10.4081/acbr.2020.106 with aggressive post-operative physiotherapy is the mainstay of management. There is paucity of reports on the presentation and management of this condition from our centre. We present two patients, 16 and 20 years old, with TMJ ankylosis in our centre. Both of them presented after 12 and 18 years, respectively, of initial injury to the TMJs.

\section{Introduction}

Ankylosis of the Temporomandibular Joint (TMJ) refers to bony or fibrous adhesion of the anatomical components of the joint and their ensuing loss of function. ${ }^{1}$ The TMJ is a unique joint because both the right and left joints must open synchronously for function, and therefore, any pathology in one or both joints results in functional problems with associated poor quality of life. ${ }^{2}$ The commonest cause of TMJ ankylosis is trauma. ${ }^{1}$ Other causes are ankylosing spondylitis, rheumatoid arthritis, psoriasis, tumours and surgeries related to the TMJ. ${ }^{3}$ TMJ ankylosis may be classified according to site (intra-capsular or extra-capsular), type of tissue involved (bony, fibrous or fibro-osseous) and the degree of fusion (complete or incomplete). ${ }^{4}$

Post-traumatic TMJ ankylosis usually develops in children after untreated condylar fracture before the age of 10 with an almost equal gender predilection. ${ }^{5}$ Retardation in the facial growth, and consequently problem with appearance, mastication, poor oral hygiene complicated with rampant caries, swallowing, digestion, and speech are often associated with TMJ ankylosis. ${ }^{6}$ Facial asymmetry is seen if there is unilateral affectation of the joint. ${ }^{6}$ The restricted facial and mandibular growth with a resulting airway insufficiency could lead to physical and psychological disability. ${ }^{2}$ Generally, the development of these associated features occurs over time, ranging from several months to decades after the initial injury. 4

Management of TMJ ankylosis is related to the associated features, the extent of the joint pathology, which also depends on the age of the patient or the time lapse since the precipitating injury occurred. ${ }^{7}$ The techniques of treatment that have been described include gap arthroplasty, interpositional arthroplasty, osteotomy across, and excision of, the ankylotic mass within the TMJ with reconstruction of the ramus/condyle unit with autogenous bone or alloplastic material. ${ }^{8}$

Early presentation after the initial injury to the TMJ would mean that simple or less extensive surgical treatment will be required by the patient. ${ }^{9}$ The reality, however, is that many patients present to the surgeons, years or decades after the initial injury, and so TMJ ankylosis would have been fully established. There is 
paucity of reports of the management of this condition in our centre. Hence, we present two patients with TMJ ankylosis successful treated in our centre.

\section{Case Report}

The first case is a 16 years old male with a 12 years history of progressive limitation in mouth opening. Patient was from a family with a low economic status. The patient presented with his mother. At 4 years old, he had a fall where he landed on his chin but he did not seek medical attention owing to only moderate discomfort. The parent, however, noticed he could not open the mouth as wide as he normally did after about 6 months but this was discountenanced as they thought it would improve with time. Patient sought definitive management when he was around the age of 14 because of severe limitation in mouth opening. Owing to cost of investigations, he presented to our centre 2 years later. The patient presented with the classical 'bird face' fascie (Figure 1a) and a prominent gonial notch with little or no movement of both condyles and there is a transverse submental scar of about $4 \mathrm{~cm}$. There was an inter-incisal distance of $2 \mathrm{~mm}$ on maximum effort at mouth opening with the presence of 28 (12 lower) teeth (Figure 1-3) (1 $1^{\text {st }}$ molars, being the last lower teeth, were carious). The CT scan showed complete obliteration of the joint space with osseous tissue, Shawney type III, with bilateral elongation of the coronoid process The surgery, interpositional arthroplasty using pterygomasseteric sling was finally done in 2019, that is, after 2 years of initial presentation to our centre. The patient is currently being reviewed. He is on daily jaw exercise at home and professional jaw exercise in the clinic on an alternate day basis. The $2^{\text {nd }}$ case involves an 18 years old female with similar family and socioeconomic history. She had a trauma to the chin from a fall at 2 years old. She presented at a nearby Primary Health Centre where the wound on the chin was treated before being discharged home. Patient's mouth opening started reducing a year after but the parent did not know that something could be done to treat her condition until 12 years of age when the mother was advised by a friend to take the child to a hospital. The patient presented at our centre with similar clinical features (Figure 4-6) to the first case. The CT scan showed a malformed mandible with complete obliteration of the joint space with osseous tissue, Shawney type IV and the surgery (gap arthroplasty with interposition using pterygomasseteric sling) to open the mouth was also done.
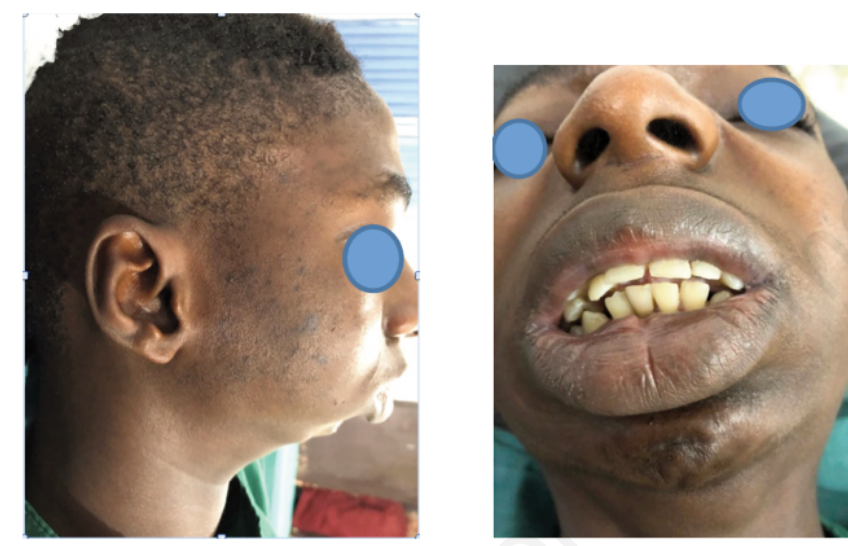

Figure 1. Preoperative photographs showing 'bird-fascie' and maximal opening of the patient.
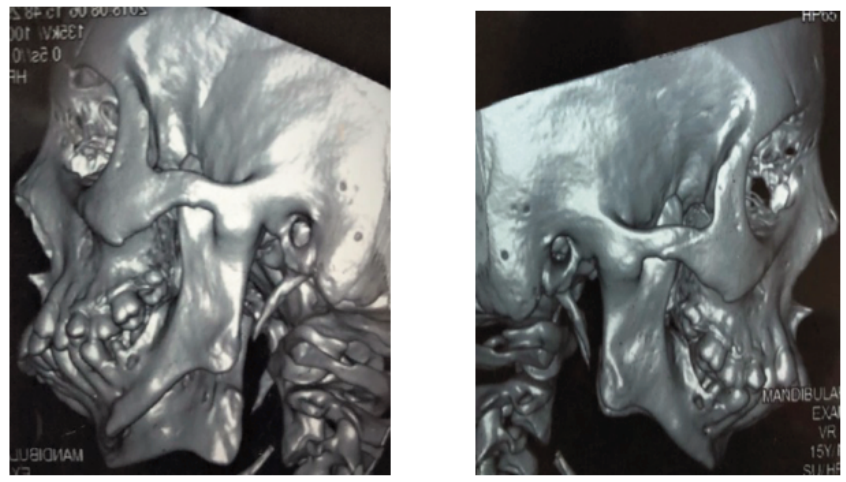

Figure 2. 3D CT showing complete fusion of the right and left joints, and also elongation of the coronoid.
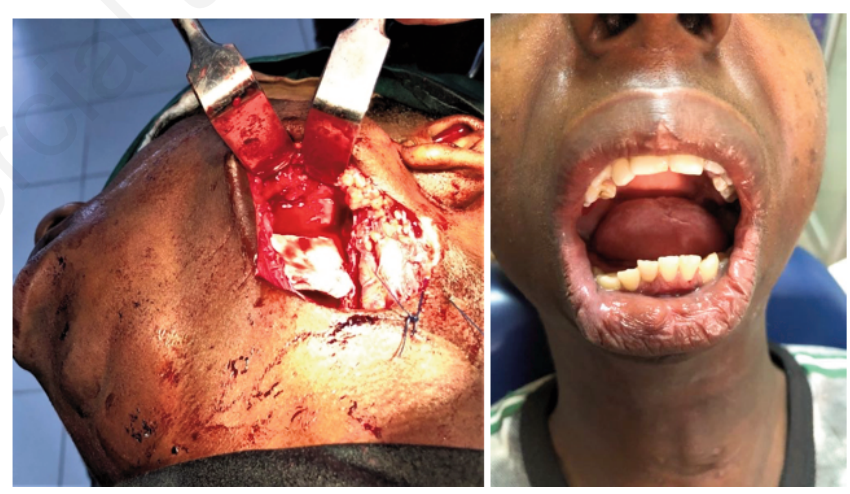

Figure 3. Intraoperative photograph showing gap arthroplasty with interposition and mouth opening after 1 year.
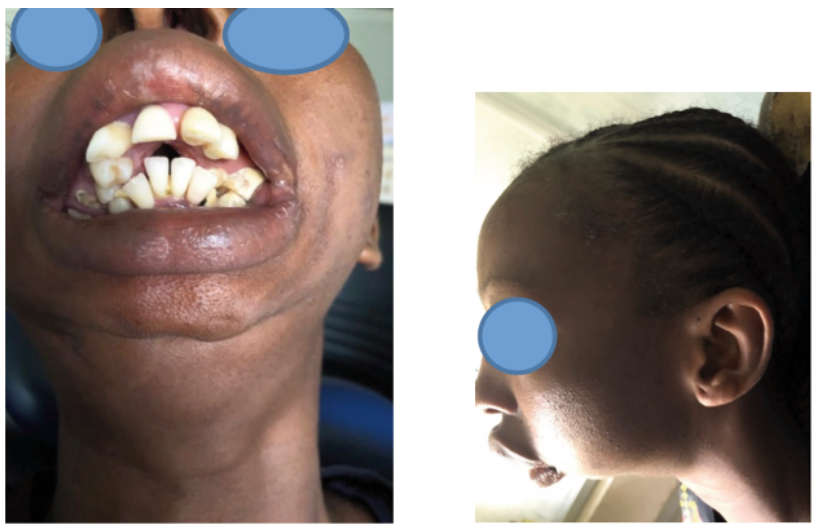

Figure 4. Preoperative photographs showing 'bird-fascie' and maximal opening of the patient. 

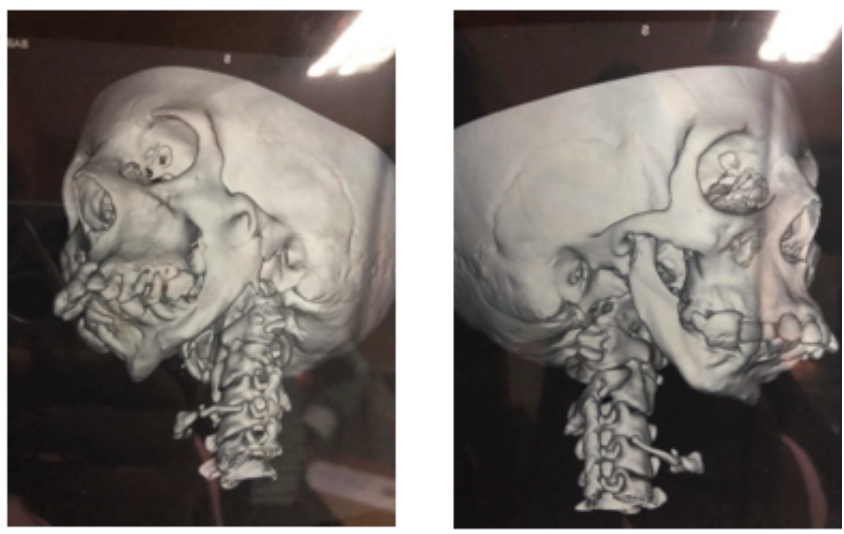

Figure 5. 3D CT showing complete fusion of the right and left joints, and also elongation of the coronoid.

\section{Discussion}

The combination of poverty, ignorance, and sometimes lack of early access to treatment have been said to result in delayed treatment of this condition. ${ }^{1}$ The two patients in this review were both affected by these factors. Nagori et al. ${ }^{9}$ particularly cited the issues of parents' low annual income and level of education as the most implicated cause of late presentation in their review of 90 patients with TMJ ankylosis. ${ }^{9}$ These two factors were also echoed by Yew et al. ${ }^{10}$ Poverty rate has been observed to reduce with the increase in literacy rate. ${ }^{11}$ With increased literacy, health-related information is known and, consequently, health-seeking behavior is improved. The opposite is also true, as pointed out by Darren and Pignone. $^{12}$

The patients claimed they presented to the primary health centre closed to them but were referred because of the non-availability of expertise. This is a big problem in Nigeria as noted by Bello et $a l .{ }^{1}$ because, the health facilities available in the country are not adequate to cater for the population, which results in people having to travel some distance, ultimately leading to delayed presentation.

One of the consequences of delayed or late presentation is that the associated features of TMJ ankylosis are well developed clinically and radiographically. The two patients show the classical bird's fascie, associated with bilateral ankylosis, also called Andy Gump or Vogelgesicht deformity, where the upper anteriors are proclined, there is severe mandibular retrognathia and retrogenia with double chin effect. ${ }^{8}$ This development complicates the treatment as orthodontic treatment and orthognathic surgery are usually indicated. ${ }^{13}$ This treatment protocol, however, was not employed in the treatment of these 2 patients because of financial challenge.

Interpositional arthroplasty with interposition using pterygomandibular sling was done for our patients. Complete resection of ankylotic mass with/without reconstruction of the ramus condyle has been said to be the ideal protocol for management of TMJ ankylosis, ${ }^{3}$ but diverse opinions exist. According to Salins ${ }^{14}$ and Malhotra et al. ${ }^{15}$ gap arthroplasty with interposition with autogenous tissue, where no resection of the ankylotic mass is done, is the treatment of choice for adults with TMJ ankylosis, where facial growth is not a concern. The rationale behind this treatment protocol is that radical removal of the bone results in large opposing surfaces of healing bone, which tend to be bridged by tough scar tissue. This scar tissue can result in restriction of mandibular movement leading to re-ankylosis. ${ }^{14}$ Also, complication, like inadvertent
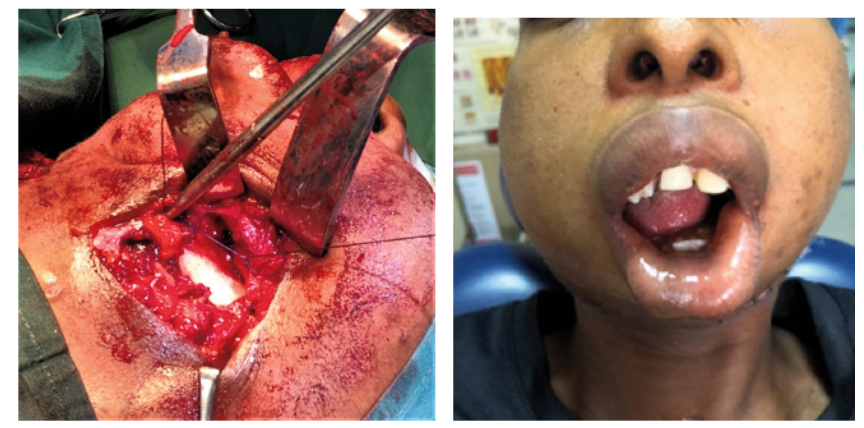

Figure 6. Intraoperative photograph showing gap arthroplasty with interposition and mouth opening after 1 year.

laceration of the sigmoid sinus, ${ }^{2}$ are frequently encountered with the complete excision of the ankylotic mass. There was not any major intraoperative and postoperative complication with the two patients.

Immediate aggressive postoperative jaw exercise is an important aspect of management of TMJ ankylosis to prevent adhesion and subsequent re-ankylosis. ${ }^{16}$ Various appliances that have been employed include 'stacking of wooden spatula', use of mason or Ferguson gag, metal or acrylic corkscrew, Therabite jaw exerciser and interocclusal splint, acrylic corkscrew among others, ${ }^{17}$ we employed the use of acrylic corkscrew for the patients, since it is easily fabricated and can be used for a wide range of mouth opening because of its shape. The postoperative mouth opening has been adequate in spite of the challenges of patient finding it difficult to come for their postoperative review.

\section{Conclusions}

Early diagnosis is crucial in prevention of the development of ankylosis, because when TMJ ankylosis is fully established an extensive surgical procedure is required to achieve an ideal mouth opening together with correction of the resultant facial deformity.

\section{Recommendations}

Every clinics and hospitals should be equipped with necessary man-power to ensure that patients do not have to travel over a long distance to seek treatment.

National Health Insurance Scheme (NHIS) is a way to make health affordable to all and sundries, and should be improved and funded robustly.

\section{References}

1. Bello SA, Aluko Olokun B, Olaitan AA, Ajike SO. Aetiology and presentation of ankylosis of temperomandibular joint: report of 23 cases from Abuja, Nigeria. Br J Oral Maxillofac Surg 2012;50:80-4.

2. Braimah R, Taiwo A, Ibikunle A, et al. Clinical experience in managing temporomandibular joint ankylosis: five-year appraisal in a Nigerian subpopulation. J Korean Assoc Oral Maxillofac Surg 2018;44:112-9. doi:10.5125/jkaoms. 


\subsubsection{2.}

3. Kaban LB, Perrott DH, Fisher K. A protocol for management of temporomandibular joint ankylosis. J Oral Maxillofac Surg 1990;48:1145-52. doi:10.1016/0278-2391(90)90529-b.

4. Chidzongham MM. Temperomandibular joint ankylosis: review of thirty-two cases. Br J Oral Maxillofacial Surg 1999;37:123-6.

5. Laskin DM. Role of the meniscus in the aetiology of post traumatic temperomandibular joint ankylosis. Int J Oral Surg 1978;7:340-5.

6. Sporniak-Tutak K, Janiszewska-Olszowska J, Kowalczyk R. Management of temporomandibular ankylosis--compromise or individualization--a literature review. Med Sci Monit 2011;17:RA111-6. doi:10.12659/msm.881755.

7. Topazian RG. Etiology of Ankylosis of Temperomandibular Joint: Analysis of 44 cases. J Oral Surg Anaesth Hosp Dental Serv 1964;22:227-33.

8. Taranjit K, Gopal K, Abhimanyu S. Factors Influencing Treatment Plan and Post-Operative Compliance in TMJ Ankylosis Patients: A Retrospective Analysis of Long Term Post-Surgical Evaluation. J Maxillofac Oral Surg 2015;14:1723.

9. Nagori SA, Jose A, Bhutia O, Roychoudhury A. Undiagnosed mandibular condylar fractures causing temporomandibular joint ankylosis: a problem in northern India. Natl Med J India 2014;27:251-5.
10. Yew CC, Rahman SA, Alam MK. Temporomandibular joint ankylosis in a child: An unusual case with delayed surgical intervention. BMC Pediatr 2015;15:169.

11. Graham, C. Mobility, opportunity and vulnerability: the dynamics of poverty and inequality in a global economy. $\mathrm{J}$ Human Develop 2002;3:57-94.

12. Dewalt DA, Pignone MP. The role of literacy in health and health Care. Am Fam Physician 2005;72:387-8.

13. Vibhute PJ, Bhola N, Borle RM. TMJ ankylosis: multidisciplinary approach of treatment for dentofacial enhancement-a case report. Case Rep Dent 2011;1-7.

14. Salins PC. New perspectives in the management of: Craniomandibular ankylosis. Int J Oral Maxillofac Surg 2000; 29:337-40.

15. Malhotra VL, Singh V, Rao JKD, et al. Lateral arthroplasty along with buccal fat pad inter-positioning in the management of Sawhney type III temporomandibular joint ankylosis. J Korean Assoc Oral Maxillofac Surg 2019;45:129-34.

16. Park MW, Young Eo MB, Yeon Seo, TTH Nguyen, SM Kim. Gap arthroplasty with active mouth opening exercises using an interocclusal splint in temporomandibular joint ankylosis patients. Maxillofacial Plastic Reconstruct Surg 2019;41:18.

17. Meredith A, Maria JT, Leonard BK. Hypomobility and hypermobility disorders of the temporomandibular joint. In: Michael M. Peterson's principles of oral and maxillofacial surgery. 4th Edition, BC Decker: 2004. Pg 1033. 